\title{
Morphogenesis of post-Golgi transport carriers
}

\author{
Alberto Luini · Alexander A. Mironov • \\ Elena V. Polishchuk · Roman S. Polishchuk
}

Accepted: 28 November 2007 / Published online: 23 January 2008

(C) Springer-Verlag 2008

\begin{abstract}
The trans-Golgi network (TGN) is one of the main, if not the main, sorting stations in the process of intracellular protein trafficking. It is therefore of central importance to understand how the key players in the TGN-based sorting and delivery process, the post-Golgi carriers (PGCs), form and function. Over the last few years, modern morphological approaches have generated new insights into the questions of PGC biogenesis, structure and dynamics. Here, we present a view by which the "lifecycle" of a PGC consists of several distinct stages: the formation of TGN tubular export domains (where different cargoes are segregated from each other and from the Golgi enzymes); the docking of these tubular domains onto molecular motors and their extrusion towards the cell periphery along microtubules; the fission of the forming PGC from the donor membrane; and the delivery of the newly formed PGC to its specific acceptor organelle. It is now important to add the many molecular machineries that have been described as operating at the TGN to this "morphofunctional map" of the TGN export process.
\end{abstract}

Keywords Golgi complex · trans-Golgi network · PostGolgi carriers · Post-Golgi transport

\section{Introduction}

The Golgi complex serves as the central station in the biosynthetic pathway, where proteins are sorted for their

A. Luini $(\bowtie) \cdot$ A. A. Mironov · E. V. Polishchuk ·

R. S. Polishchuk $(\bowtie)$

Department of Cell Biology and Oncology, Consorzio Mario Negri Sud,

66030 Santa Maria Imbaro (Chieti), Italy

e-mail: luini@ negrisud.it

e-mail: polish@negrisud.it different destinations, such as various domains of the cell surface and the endosomal-lysosomal system. This delivery of cargo proteins from the Golgi complex to their target compartments is carried out by dynamic, membrane-bound organelles that are frequently called either "transport carriers" or "post-Golgi carriers" (PGCs) (Luini et al. 2005). As these PGCs have an important role in the process of intracellular transport, their morphology, living dynamics and molecular compositions have become the focus of significant interest, particularly over the last few years.

These PGC transport organelles were originally defined through the development of green fluorescent protein (GFP) technology and living-cell imaging (Lippincott-Schwartz et al. 2000), whereby the first fluorescent cargo protein that was followed in living cells revealed a new world of images of these relatively large and highly dynamic structures that travel from the Golgi complex to the plasma membrane (Wacker et al. 1997; Hirschberg et al. 1998; Nakata et al. 1998). Then, with time, the list of cargo molecules that could be visualized in vivo expanded, further exposing the unexpected complexity of the post-Golgi transport pathways. Finally, further technical advances resulted in the combination of video and electron microscopy (correlative light-electron microscopy, CLEM), which provided the means to define the morphogenesis of these PGCs at the ultrastructural level.

As a result of these studies we now know that PGCs arise from specific membrane domains of the Golgi complex that lack resident Golgi enzymes, forming what are known as the "PGC precursors" (Hirschberg et al. 1998; Keller et al. 2001; Polishchuk et al. 2003; Puertollano et al. 2003). The shapes and sizes of PGCs that even carry the same cargo can vary across a wide range, and under the light microscope, most of them are seen to be clearly larger that plasma-membrane associated clathrin vesicles and 
100-nm fluorescent beads (Hirschberg et al. 1998). The smaller PGCs usually have a size of 300-400 nm, although some of the larger ones can reach several microns in length. Video microscopy has also revealed that many of these carriers appear globular, although they frequently stretch out into tubular shapes during their translocation through the cytosol; thus PGCs have frequently been termed as "pleiomorphic" structures.

In transporting their cargoes to the correct acceptor compartments, the movement of PGCs is mediated via microtubules. However, carriers can also form and support postGolgi transport without microtubules, although the correct targeting of their cargo proteins is usually compromised under these conditions (Rindler et al. 1987; Kreitzer et al. 2003). Finally, the life cycle of a PGC can be schematically imagined to consist of three stages: (1) its formation (which can in turn be further divided into several substages; see below); (2) its transition through the cytosol; and (3) its docking and fusion with the target membrane (Polishchuk et al. 2000). The first of these steps, the formation of the PGC, appears to be the most complex, and it also probably remains the least understood.

Since the question of the morphogenesis of a PGC is closely intertwined with that of the structure of the organelle from which it originates, the trans-Golgi network (TGN), both of these issues will now be discussed in an integrated fashion.

\section{The trans-Golgi network and the origin of PGCs}

The process of PGC morphogenesis has been characterized in detail using video and electron microscopy, as well as with the combination of these techniques (CLEM). As indicated above, this process comprises the formation, the extrusion and the fission of the export domain from the TGN, thus generating the free carrier (Polishchuk et al. 2003; see also Fig. 1). The first step (the formation of the tubular export domains) also includes the segregation of the cargo proteins from the Golgi resident enzymes. These events appear to be common to the proteins directed towards different post-Golgi compartments, such as basolateral plasma membrane (Hirschberg et al. 1998; White et al. 2001; Polishchuk et al. 2003), the apical plasma membrane (Keller et al. 2001) and the endosomal-lysosomal system (Puertollano et al. 2003). These export domains usually contain TGN markers; i.e. they are part of the TGN (Polishchuk et al. 2003; Puertollano et al. 2003). Of note, these three post-Golgi compartments are the ones that have been best characterized for the PGCs leaving the TGN, with the total number and types of pathways for cargo exit from the TGN at present not known, although there are at least six, and probably more (Rodriguez-Boulan et al. 2005).
Although the existence of the TGN has been known for many years (Griffiths and Simons 1986), its precise structure and identity still remain to be ascertained. In one view, which predominated in the field until recently, the TGN was considered to consist of essentially an anastomosing tubular network that emanates from (or that results from the breakdown of) the trans-most Golgi cisterna, and that projects mainly in the trans direction (Griffiths et al. 1989, 1985; Clermont et al. 1995). Rambourg and colleagues have thoroughly described the TGN in several different cell types. In these descriptions, this trans-most cisterna also tends to "peel off" from the rest of the Golgi stack, and together with the other morphological characteristics of the Golgi stack, this is a feature that is suggestive (Clermont et al. 1995) of the cisternal progression-maturation trafficking model (Bannykh and Balch 1997; Mironov et al. 1997; Bonfanti et al. 1998; Losev et al. 2006; Matsuura-Tokita et al. 2006). Thus, in a simple version of this scheme, the TGN would result from the final stages of maturation of the Golgi cisternae. This would include the partial transformation of this trans-most cisternae into a tubular network, and the various carriers that leave the TGN for their different destinations would all originate form different domains of this tubular network.

A more complex and more recent view of the organization at the trans face of the Golgi complex comes from studies using electron microscopy tomography of cryofixed, freeze-substituted cells (Ladinsky et al. 1999, 2002). These studies have indicated that the TGN derives not only from the last trans cisterna, but instead from the three trans-most cisternae (Roth et al. 1985), from where tubules emanate into the trans space of the Golgi stacks. Remarkably, only the trans-most cisterna exhibits clathrin-coated buds, with the other buds appearing to have different, yetto-be identified, types of coats (Ladinsky et al. 1999). Thus, only the trans-most cisterna would be responsible for the clathrin- and AP-1-dependent trafficking towards the endolysosomes, and although all of these three trans-most cisternae of the Golgi stacks would serve as the classical TGN, each of them could be specialized in the packaging and export of specific cargo proteins. This might have important functional and mechanistic implications for the proteins sorting and export process.

Another remarkable TGN feature that has been revealed by these tomographic studies is that these three trans-most cisternae can intercalate with the cisternae of the endoplasmic reticulum (ER) (Ladinsky et al. 1999, 2002). These have been proposed to have important roles in the transfer of lipids (through specialized contact sites) between the ER itself and the Golgi complex.

It will be important to establish whether the morphological discrepancies described above reflect fundamental differences in the organization of the export of cargo in 
Fig. 1 Formation of post-Golgi transport carriers. Subsequent frames extracted from a timelapse sequence illustrating the different stages of PGC biogenesis (arrows): a formation of the tubular domain containing the cargo VSVG-YFP and devoid of the Golgi-resident protein galatosyltransferase-CFP. b Extrusion of this tubular domain from the Golgi complex. c, d Fission of the domain from the parental Golgi membranes. e Thin section of a cell expressing the TGN38-HRP construct. Arrows indicate tubular PGC precursor that has been pulled out of the TGN area of the Golgi complex
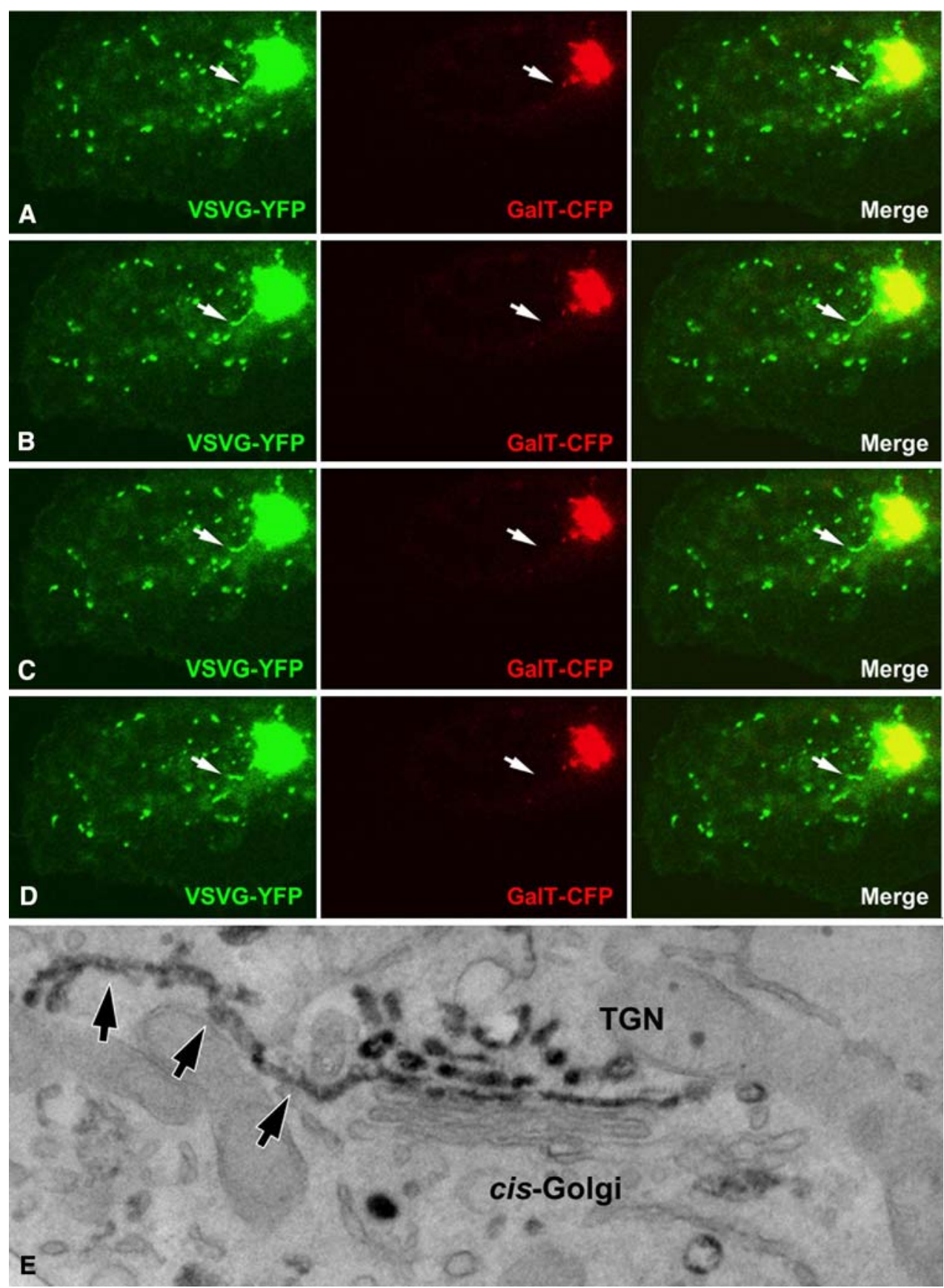

different cell systems. Indeed, it is possible that the first model (the TGN as a network resulting from the tubular disassembly of the last trans-most cisterna) might actually only represent a special case of the more complete threetrans-cisterna model, and that both of these two different models of TGN may apply, depending on the cell type and the functional conditions.

In support of this possibility, it is clear that the TGN can vary significantly in both size and composition across different cell types. For example, cells with a well-developed endo-lysosomal system and without secretory granules exhibit an extensive tubular TGN, while the tubular component of the TGN is reduced in cells that are specialized in regulated secretion (Clermont et al. 1995). This appears to occur in the latter because most of the TGN membranes are used for the packaging of secretory granules, and the tubules only form the thin bridges between the granule precursors. Such an organization simplifies the release of the granules, which occurs through the rupture of these tubular elements (Clermont et al. 1995). Similarly, human fibroblasts have large collagen-containing distensions that are connected to tubular elements in the transGolgi area (Polishchuk et al. 2003). Thus, the morphology and size of the TGN apparently depend on the predominant 
type and amount of cargo protein departing from the Golgi complex.

The morphology of the TGN depends also on its secretory status. When exit from the TGN is blocked by a lowering of the temperature to $20^{\circ} \mathrm{C}$ in the presence of abundant cargo (Matlin and Simons 1983), the volume and surface areas of the TGN increase greatly (Griffiths et al. 1989); while in the absence of cargo, the $20^{\circ} \mathrm{C}$ block results in all three of the trans-most cisternae producing bulging exit domains (Ladinsky et al. 2002). At physiological temperatures, three-dimensional (3D) analyses of the Golgi stacks have revealed well developed, tubular-reticular, TGN-like membranes at the trans side of the Golgi complex in actively secreting cells (Trucco et al. 2004), while the TGN is nearly absent in quiescent cells (Trucco et al. 2004). Thus, the TGN is a very dynamic structure, the shape and size of which is potently regulated by the extent of traffic flowing through it. To discover the regulatory mechanisms is an important challenge for the future.

Another challenge will be to define whether the threecisterna organization really underlies a functional specialization of these cisternae in the sorting and packaging of different cargo classes, as suggested by the presence of clathrin buds only on the last cisterna of the Golgi stack. So far, the lack of immunolabeling studies does not allow determining whether different trans cisternae really do contain different transport proteins.

In addition to being an organelle of the biosynthetic pathways, the TGN is involved in endocytic transport routes (Griffiths and Simons 1986; Mellman and Simons 1992; Pavelka et al. 1998). Several studies with different cell types showed that the TGN and Golgi stacks can contain plasma-membrane constituents and internalized materials (Stoorvogel et al. 1988; van Weert et al. 1997). These exchanges with the endocytic systems could well introduce a further level of structural complications, since the recycling endosomes, in particular, reside in the Golgi area and are tubular in nature. In conclusion, both the dynamics and the structure of the TGN are variable and incompletely defined, thereby presenting an additional layer of difficulty to our understanding of the biogenesis of the carriers that depart from this organelle.

\section{Formation of PGC precursors at the TGN}

There are two key aspects to the formation of the PGC precursors: their morphogenesis at the TGN; and the segregation between different types of cargo proteins that are targeted for different destinations, as well as between the Golgi-resident proteins, such as the Golgi enzymes.

Regarding PGCs morphogenesis, in principle, the tubular carrier precursors might form through the mechanical pulling force exerted by microtubule-based motors on a flatter, parent, membrane domain (e.g., a Golgi cisterna). This has been shown to be mechanistically possible for both artificial and natural membranes (Roux et al. 2002). If this is the case, the PGC precursors would be expected to be essentially simple linear tubular structures (Roux et al. 2002). The second possibility is that these precursors are actually formed from the tubular subdomains of the TGN, which are generated prior to docking and extrusion by microtubules. In this case, the GPCs should reflect the morphologically complex structure of their parent membranes.

Recent studies have provided compelling support in favor of the latter model (Polishchuk et al. 2003). First, both free PGCs and their precursors at the TGN comprise complex tubulo-reticular structures, which have often been described as the main component of the TGN in many cell types (Clermont et al. 1995). For example, PGCs carrying VSVG show a mostly tubular morphology, and can have a complex structure that even contains clearly visible fenestrae (Polishchuk et al. 2003); this would be expected of membranes that derive from protrusions of the TGN. Indeed, PGC precursors that have been visualized using CLEM, appear to be comprised of tubular segments that are interconnected with complex branching and fenestrated membranes; they are also seen to be continuous with the parent membranes of the Golgi stack (Polishchuk et al. 2003). Similarly, carriers containing the apical cargo protein hemagglutinin (HA) frequently have a tubular morphology as well as HA-positive domains at the TGN (Puertollano et al. 2001). Thus structural similarities between PGC precursors and the TGN appear to be a common feature of different types of PGCs. This strongly suggests that PGCs form via the fission of an entire precursor domain (or a large part thereof) from the rest of the TGN membranes.

Thus the question regarding the mechanism by which the originally flat Golgi membranes are converted into highly bent, tubular-reticular TGN structures arises. Essentially, this can be achieved by one of two mechanisms: either by the action of proteins that can bend these membranes into tubules, in a manner that is possibly similar to that of amphiphysin, endophilin, sorting nexins and others (Antonny 2006), or via the alteration of the lipid composition of these trans-Golgi membranes, which can be mediated, in turn, by several processes. For instance, changes in lipid content, and hence membrane curvature, can be modulated via the lipid-metabolizing enzymes that reside at the Golgi complex (for review, see De Matteis and Godi 2004; Luini et al. 2005). The transmembrane or inter-organelle transfer of lipids can contribute to the generation of particular lipid environments in the membranes of the TGN. In this respect, it is important to note that numerous contact sites between the ER and the trans-cisternae of the Golgi 
complex have been detected by electron microscopy tomography (Ladinsky et al. 1999, 2002). Such contact sites can be explored for lipid transfer between the ER and trans-Golgi that is mediated by specific lipid-transfer proteins. Also, since the cisterna-like morphology of Golgi compartments can be stabilized by large polymers of Golgi enzymes (Nilsson et al. 1996), the loss of oligomerized Golgi enzymes in the trans-Golgi should, in principle, favor cisterna-to-tubule transformation of cargo-containing membranes. Moreover, this process can be accompanied by the loss of the stacking mechanisms at the trans side of the Golgi complex. Here, GRASP65 and GRASP55 are two proteins that have been suggested to be involved in the maintenance of cisterna juxtapositioning, and they are located mainly at the cis and medial Golgi, rather than the trans-Golgi (Barr et al. 1997; Shorter et al. 1999). So this intercisternal "glue" may be gradually lost as a cisterna progresses towards the trans pole of the Golgi complex. This has been confirmed both in mammals and yeast by the observation that the trans-most cisterna frequently peels off from the main Golgi stack (Clermont et al. 1995; Mogelsvang et al. 2003). It is possible that more than two or more mechanisms act in synergy to provide this transition from flat Golgi cisternae to the tubulo-reticular TGN morphology.

In addition to the formation of tubular domains at the exit face of the Golgi complex, this is also the level at which cargo proteins that are directed to different postGolgi destinations should be sorted. The classical view in the membrane transport field implies that sorting at the TGN (as well as throughout the whole secretory pathway) is driven mainly by the coat-adaptor-protein machinery, which interacts specifically with amino-acid signals of certain transmembrane cargo proteins; this then provides the mechanical force for budding and fission of transport vesicle (Mellman and Warren 2000).

This holds true for the endo-lysosome-directed carriers that have been thoroughly characterized. These carriers consist of clusters of clathrin-coated buds that are connected by tubular regions, and thus exhibit a grape-like structure. In contrast, PGCs carrying a cargo like the G-protein of vesicular stomatitis virus (VSVG) form in a coat and an AP-independent manner. Both PGCs and their precursors do not show $\beta$-COP or $\gamma$-, $\delta$ - and $\varepsilon$-adaptins at their membranes (Polishchuk et al. 2003). Other adaptors, such as the GGAs, are excluded from VSVG carriers as well (Puertollano et al. 2003; Polishchuk et al. 2003). Similarly, coats and adaptors have also never been detected on PGCs that are carrying proteins to the apical surface in polarized cells (Kreitzer et al. 2003). Thus, these carriers should form either by virtue of some still-unknown adaptors that cannot yet be visualized by electron microscopy, or by their association with specific lipid microdomains that are involved in sorting (Schuck and Simons 2004). This might be the case for proteins directed to the apical surface in polarized epithelial cells, the concentration of which at the TGN appear to be through their partition into cholesterol- and sphingolipid-rich membrane domains that are known as "rafts" (Schuck and Simons 2004).

\section{The scission of PGCs from the TGN}

The morpho-dynamics of the fission process are also fairly complex. Observations in living cells have revealed that the fission of PGCs frequently coincides with mechanical pulling of carrier precursor from the TGN along microtubules. Apparently, the pulling force which the molecular motors such as kinesin (see below) can apply to the TGN membranes is important to facilitate the extension of PGC precursors from the Golgi body and for the later fission of the PGC (Kreitzer et al. 2000; Polishchuk et al. 2003). In cellfree systems, the addition of kinesin to Golgi membranes (and even to liposomes) together with microtubules induces the formation of tubule-like membranes that are similar to PGC precursors (Roux et al. 2002), while a block of kinesin function by microinjection of an inhibitory antibody (Kreitzer et al. 2000) or expression of the headless kinesin mutant (Nakata and Hirokawa 2003) prevent PGC formation from the Golgi complex. Kinesin has been seen to be associated with the tip of PGC precursors, although it can also attach to other points along the PGC precursor membrane (Polishchuk et al. 2003). The movement of kinesins along microtubules can then create tension within the PGC precursor that will facilitate the fission process. Indeed, based on in vitro data, membranes under tension have recently been proposed to have an important role in fission (Roux et al. 2006). However, PGCs can also form when microtubules have been destroyed by nocodozole treatment; in this case, the pulling force to create membrane tension in fissionprone regions might be provided by actin motors (Warner et al. 2003; Sahlender et al. 2005).

Live cell imaging and CLEM have also shown that fission does not take place randomly along the membranes of PGC precursors. In this case, which regions of a PGC precursor can be defined as prone to fission? First, this depends on the geometry of the precursor membranes. Our data suggest that fission usually takes place at the thinnest parts of the PGC precursor (Fig. 2a), which at the electron microscopy level corresponding to thin tubular segments of membranes (Polishchuk et al. 2003). In contrast, fission does not take place at the TGN regions with a complex morphology (i.e., in those containing tubular networks and branching tubules, or in thick vacuolar regions). Obviously, the precise points of fission will define not only the compositions of the PGC carriers, but also their morphology. 

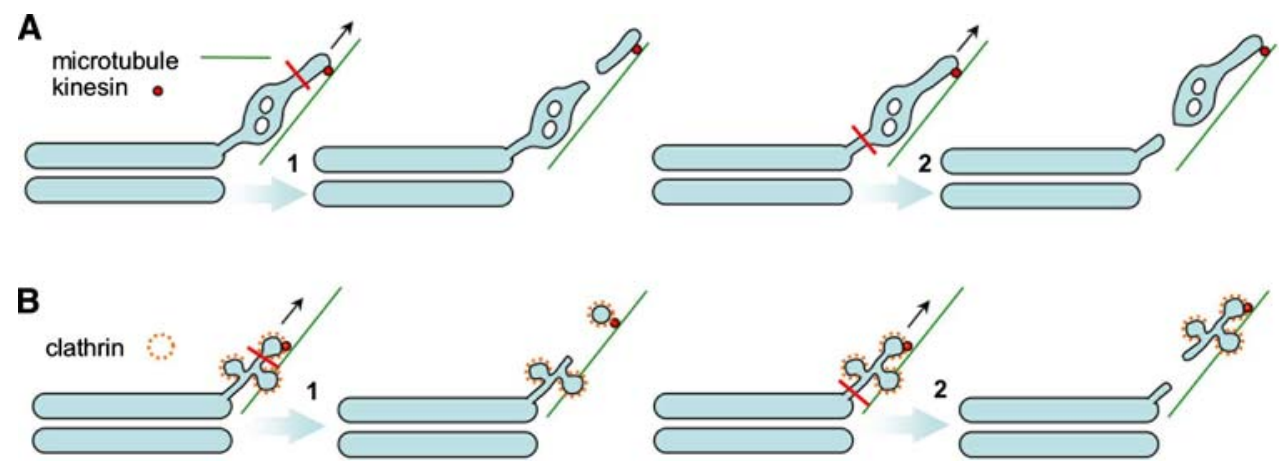

Fig. 2 Fission of post-Golgi transport carriers. a TGN precursors of post-Golgi carriers are pulled along microtubules by kinesin. The fission (red line) of the carriers occurs at the thinnest parts of the PGC precursor, which correspond to thin tubular segments of the TGN membrane at the electron microscopy level. In contrast, fission does not take place at the TGN regions with a complex morphology (i.e., containing tubular networks and fenestrae, or in thick vacuolar re-

If fission occurs close to the tip of the TGN tubule, a carrier will be smaller in size. In contrast, larger PGCs can form by cleavage at the base of the PGC precursors (Fig. 2a). Similarly, endosome-directed PGCs can apparently detach from the TGN as simple cargo-containing vesicles if the fission occurs at the neck of the clathrin-coated buds (Fig. 2b). However, many clathrin-positive PGCs have a grape-like morphology (tubule with several buds), suggesting that entire chunks of TGN membranes containing $2-3$ clathrincoated buds can be cleaved from the Golgi complex (Polishchuk et al. 2006).

Another factor that might be important for PGC shaping is the type of cargo that is embedded in the PGC. For example, procollagen-I usually forms quite large aggregates that are visible within 300-400-nm-diameter membrane distensions of the Golgi membranes. As a consequence, similar distensions have been detected in most collagen-containing PGCs (Polishchuk et al. 2003; Canty et al. 2004).

\section{Movement of PGCs from the Golgi complex to the target membrane}

After fission from the TGN, PGCs move to the acceptor membrane. Different members of the kinesin superfamily (Kamal et al. 2000; Nakata and Hirokawa 2003; Teng et al. 2005), and also the other microtubule motor dynein (Tai et al. 1999), have been shown to drive post-Golgi transport of specific cargo to various destinations. This high fidelity of cargo selection by molecular motors at the TGN and their further delivery to the correct surface or intracellular domain may be regulated by interactions of motor protein directly with the cargo (Kamal et al. 2000; Teng et al. 2005) or with components of the sorting machinery at the TGN (Nakagawa et al. 2000). gions). If fission occurs close to the tip of a PGC precursor, the carrier will be smaller in size (1). In contrast, larger PGCs can be formed by cleavage at the bottom of a PGC precursor (2). b PGCs directed to endosomes detach from the TGN as simple clathrin-coated vesicles if fission (red line) occur at the neck of the clathrin-coated bud (1). Alternatively, entire chunks of the TGN membrane containing 2-3 clathrin-coated buds can be cleaved from the Golgi complex

As an example, transport of HA and annexin $13 \mathrm{~b}$ to the apical surface in epithelial cells relies on raft-associated motor KIFC3 (Noda et al. 2001). KIF13A operates in the other post-Golgi route that is used for the transport of the mannose-6-phosphate receptor (Nakagawa et al. 2000). A number of neuronal proteins, including bAPP, GAP43 and vamp-2, require KIF5 for their correct targeting (Nakata and Hirokawa 2003), while the microtubule minus-enddirected motor dynein has been shown to support rhodopsin transport in rod photoreceptors (Tai et al. 1999; Yeh et al. 2006).

Selection of specific cargoes by motors could be driven by various mechanisms. The simplest would use a direct interaction between the motor and a specific domain of a cargo protein, as is seen for the dynein light chain and the cytoplasmic tail of rhodopsin (Tai et al. 1999). Alternatively, adaptor proteins could serve as a bridge between a motor and its cargo. For example, KIF13A transports the mannose-6-phosphate receptor through its interaction with the AP-1 complex (Nakagawa et al. 2000). Finally, both motor and cargo could associate with the same specific lipid microdomain, as for instance, for KIFC3 and the apically targeted annexin XIIIb.

Another issue that needs to be addressed is whether any of these sorting processes take place in the PGCs while they are moving toward their acceptor compartment. This happens, for example, with the maturation of secretory granules when the mannose-6-phosphate receptor is concentrated and sorted from the secretory granules by clathrin-coated vesicles (Klumperman et al. 1998). So several strategies have been used to determine whether similar sorting events happen with PGCs. Mature exocytic carriers can be arrested before their fusion with the plasma membrane either by microinjection of an anti-NSF antibody or by treatment with tannic acid (which fixes the plasma 
membrane but does not penetrate inside the cell). In contrast to secretory granules, the comparison of mature and newly formed PGCs did not reveal significant changes in either their ultrastructure or their composition (Polishchuk et al. 2003, 2004). Similarly, mature Golgi-to-endosome carriers were accumulated in cells upon endosome inactivation. However, they did not show any significant transformation, except for a very moderate reduction in the area covered by clathrin (Polishchuk et al. 2006).

Live-cell imaging of subconfluent MDCK cells has shown that PCGs that initially contained both a basolateral marker (VSVG-CFP) and an apical marker (GPI-YFP) did not sort out either of these cargoes into any separate structures, and instead delivered both of the proteins to the plasma membrane (Polishchuk et al. 2004). GPI-GFP was then sorted from the basolateral surface to the apical membrane through transcytosis (Polishchuk et al. 2004). On the other hand, the partitioning of two proteins from a common PGC into separate carriers has also been reported (Jacob and Naim 2001). This suggests that sorting from the PGC may exist, but that it should depend on the nature of the cargo proteins being transported.

\section{Intermediate stations in post-Golgi transport}

The complexity of sorting events in the post-Golgi space appears to be even worse since the discovery that certain cargoes may pass through the endosomal compartments before their arrival at the plasma membrane. Sporadic reports in the past have suggested that some secretory proteins do not move from the Golgi complex directly to the plasma membrane, but instead pass through an endocytic intermediate on their way to the cell surface (Leitinger et al. 1995). This indirect "through-endosome" delivery of cargo to the plasma membrane might be also facilitated by the intimate association of TGN membranes with number of endocytic compartments in the perinuclear area of the cell (Marsh et al. 2001). The list of the proteins using this pathway has been recently updated, and it has now been shown that in MDCK epithelial cells, VSVG, the LDL receptor and E-cadherin can be detected in the endosomes before their exit to the plasma membrane (Ang et al. 2004; Lock and Stow 2005).

These findings, however, promote a number of further questions. The first is whether this transport route is ubiquitous (i.e., does it exist in different cells?). The second question is whether different cargoes move through the same endosomal compartment on their way to the cell surface in epithelial cells? A number of proteins (such as VSVG and the LDL receptor) have been reported to use a Rab8-positive sub-population of endosomes as an intermediate station on their way to the basolateral membrane in epithelial cells
(Ang et al. 2003). These proteins require the AP-1B adaptor complex to be properly sorted from these Rab8 endosomes towards the basolateral surface domain (Ang et al. 2003). Other cargoes (such as E-cadherin, for example) move to the plasma membrane through a Rab11 endocytic compartment (Lock and Stow 2005). It remains to be determined, however, whether there is any cross-talk between these Rab8- and Rab11-dependent routes. This possibility apparently exists, since Rab8 and Rab11 endosomes are both accessible to transferrin (Ang et al. 2004; Lock and Stow 2005).

Finally it is important to clarify as to what extent this through-endosome transport route is used by different cargo proteins in epithelial cells. This issue has been partially addressed by the silencing of the $\mu 1 \mathrm{~B}$ subunit of the AP- $1 \mathrm{~B}$ adaptor complex, which resides at the endosomes and is required for the correct delivery of many basolateral proteins. The use of RNA interference has revealed, however, that a number of cargoes (such as transferrin and Fc receptors, for example) can be targeted correctly even in the absence of AP-1B, which suggests that these proteins can move directly from the Golgi complex to the basolateral surface without crossing the endocytic routes (Gravotta et al. 2007). The existence of more than one basolateral pathway has also been supported by the observation that transport of different basolateral proteins can be selectively regulated by different PKD isoforms (Yeaman et al. 2004). Thus, further efforts need to be made to understand to what extent an endocytic post-Golgi compartment is involved in the sorting and transport events of cell-surface proteins.

\section{Conclusions}

The extensive characterization of PGC morphology by video and electron microscopy has provided a framework for the positioning of the molecular machineries in the morpho-functional maps of various trafficking segments. At the same time, many molecular players in TGN-to-plasma membrane transport have been identified. The attribution of each of the molecular components to each of the pathways is probably now the main challenge. We believe that the development of specific assays will significantly assist in the achieving of this objective. Video microscopy of GFPtagged cargo proteins has allowed us to evaluate the process of PGC formation, as well as the speed and directionality of PGC movement through the cytosol. Other assays use tannic acid treatment (Polishchuk et al. 2004; Jakob et al. 2006) or horse-radish-peroxidase-based endosome immobilization (Ang et al. 2004; Polishchuk et al. 2006) to prevent the fusion of PGCs with their target membranes. These conditions allow us to trap mature PGCs and to compare their compositions to newly formed carriers and 
the TGN membranes. Moreover, preferential sites of PGC docking and fusion can also be easily identified in this way. The combination of these experimental approaches with specific molecular inhibitors now allows us to attribute a protein of interest to one of the steps in post-Golgi transport.

\section{References}

Ang AL, Fölsch H, Koivisto UM, Pypaert M, Mellman I (2003) The Rab8 GTPase selectively regulates AP-1B-dependent basolateral transport in polarized Madin-Darby canine kidney cells. J Cell Biol 163:339-350

Ang AL, Taguchi T, Francis S, Folsch H, Murrells LJ, Pypaert M, Warren G, Mellman I (2004) Recycling endosomes can serve as intermediates during transport from the Golgi to the plasma membrane of MDCK cells. J Cell Biol 167:531-543

Antonny B (2006) Membrane deformation by protein coats. Curr Opin Cell Biol 18:386-394

Bannykh SI, Balch WE (1997) Membrane dynamics at the endoplasmic reticulum-Golgi interface. J Cell Biol 138:1-4

Barr FA, Puype M, Vandekerckhove J, Warren G (1997) GRASP65, a protein involved in the stacking of Golgi cisternae. Cell 91:253-262

Bonfanti L, Mironov AA Jr, Martínez-Menárguez JA, Martella O, Fusella A, Baldassarre M, Buccione R, Geuze HJ, Mironov AA, Luini A (1998) Procollagen traverses the Golgi stack without leaving the lumen of cisternae: evidence for cisternal maturation. Cell 95:993-1003

Canty EG, Lu Y, Meadows RS, Shaw MK, Holmes DF, Kadler KE (2004) Coalignment of plasma membrane channels and protrusions (fibripositors) specifies the parallelism of tendon. J Cell Biol 165:553-563

Clermont Y, Rambourg A, Hermo L (1995) Trans-Golgi network (TGN) of different cell types: three-dimensional structural characteristics and variability. Anat Rec 242:289-301

De Matteis MA, Godi A (2004) Protein-lipid interactions in membrane trafficking at the Golgi complex. Biochim Biophys Acta 1666:264-274

Gravotta D, Deora A, Perret E, Oyanadel C, Soza A, Schreiner R, Gonzalez A, Rodriguez-Boulan E (2007) AP1B sorts basolateral proteins in recycling and biosynthetic routes of MDCK cells. Proc Natl Acad Sci USA 104:1564-1569

Griffiths G, Simons K (1986) The trans Golgi network: sorting at the exit site of the Golgi complex. Science 234:438-443

Griffiths G, Pfeiffer S, Simons K, Matlin K (1985) Exit of newly synthesized membrane proteins from the trans cisterna of the Golgi complex to the plasma membrane. J Cell Biol 101:949-964

Griffiths G, Fuller SD, Back R, Hollinshead M, Pfeiffer S, Simons K (1989) The dynamic nature of the Golgi complex. J Cell Biol 108:277-297

Hirschberg K, Miller CM, Ellenberg J, Presley JF, Siggia ED, Phair RD, Lippincott-Schwartz J (1998) Kinetic analysis of secretory protein traffic and characterization of Golgi to plasma membrane transport intermediates in living cells. J Cell Biol 143:1485-1503

Jacob R, Naim HY (2001) Apical membrane proteins are transported in distinct vesicular carriers. Curr Biol 11:1444-1450

Jakob V, Schreiner A, Tikkanen R, Starzinski-Powitz A (2006) Targeting of transmembrane protein shrew-1 to adherens junctions is controlled by cytoplasmic sorting motifs. Mol Biol Cell 17:3397-3408

Kamal A, Stokin GB, Yang Z, Xia CH, Goldstein LS (2000) Axonal transport of amyloid precursor protein is mediated by direct binding to the kinesin light chain subunit of kinesin-I. Neuron 28:449459
Klumperman J, Kuliawat R, Griffith JM, Geuze HJ, Arvan P (1998) Mannose 6-phosphate receptors are sorted from immature secretory granules via adaptor protein AP-1, clathrin, and syntaxin 6positive vesicles. J Cell Biol 141:359-371

Keller P, Toomre D, Diaz E, White J, Simons K (2001) Multicolour imaging of post-Golgi sorting and trafficking in live cells. Nat Cell Biol 3:140-149

Kreitzer G, Marmorstein A, Okamoto P, Vallee R, Rodriguez-Boulan E (2000) Kinesin and dynamin are required for post-Golgi transport of a plasma-membrane protein. Nat Cell Biol 2:125-127

Kreitzer G, Schmoranzer J, Low SH, Li X, Gan Y, Weimbs T, Simon SM, Rodriguez-Boulan E (2003) Three-dimensional analysis of post-Golgi carrier exocytosis in epithelial cells. Nat Cell Biol 5:126-136

Ladinsky MS, Mastronarde DN, McIntosh JR, Howell KE, Staehelin LA (1999) Golgi structure in three dimensions: functional insights from the normal rat kidney cell. J Cell Biol 144:1135-1149

Ladinsky MS, Wu CC, McIntosh S, McIntosh JR, Howell KE (2002) Structure of the Golgi and distribution of reporter molecules at 20 degrees $\mathrm{C}$ reveals the complexity of the exit compartments. Mol Biol Cell 13:2810-2825

Leitinger B, Hille-Rehfeld A, Spiess M (1995) Biosynthetic transport of the asialoglycoprotein receptor $\mathrm{H} 1$ to the cell surface occurs via endosomes. Proc Natl Acad Sci USA 92:10109-10113

Lippincott-Schwartz J, Roberts TH, Hirschberg K (2000) Secretory protein trafficking and organelle dynamics in living cells. Annu Rev Cell Dev Biol 16:557-589

Lock JG, Stow JL (2005) Rab11 in recycling endosomes regulates the sorting and basolateral transport of E-cadherin. Mol Biol Cell 16:1744-1755

Losev E, Reinke CA, Jellen J, Strongin DE, Bevis BJ, Glick BS (2006) Golgi maturation visualized in living yeast. Nature 441:10021006

Luini A, Ragnini-Wilson A, Polishchuck RS, De Matteis MA (2005) Large pleiomorphic traffic intermediates in the secretory pathway. Curr Opin Cell Biol 17:353-361

Marsh BJ, Mastronarde DN, Buttle KF, Howell KE, McIntosh JR (2001) Organellar relationships in the Golgi region of the pancreatic beta cell line, HIT-T15, visualized by high resolution electron tomography. Proc Natl Acad Sci USA 98:2399-2406

Matlin KS, Simons K (1983) Reduced temperature prevents transfer of a membrane glycoprotein to the cell surface but does not prevent terminal glycosylation. Cell 34:233-243

Matsuura-Tokita K, Takeuchi M, Ichihara A, Mikuriya K, Nakano A (2006) Live imaging of yeast Golgi cisternal maturation. Nature 441:1007-1010

Mellman I, Simons K (1992) The Golgi complex: in vitro veritas? Cell 68:829-840

Mellman I, Warren G (2000) The road taken: past and future foundations of membrane traffic. Cell 100:99-112

Mironov AA, Weidman P, Luini A (1997) Variations on the intracellular transport theme: maturing cisternae and trafficking tubules. J Cell Biol 138:481-484

Mogelsvang S, Gomez-Ospina N, Soderholm J, Glick BS, Staehelin LA (2003) Tomographic evidence for continuous turnover of Golgi cisternae in Pichia pastoris. Mol Biol Cell 14:2277-2291

Nakagawa T, Setou M, Seog D, Ogasawara K, Dohmae N, Takio K, Hirokawa N (2000) A novelmotor, KIF13A, transports mannose6-phosphate receptor to plasma membrane through direct interaction with AP-1 complex. Cell 103:569-581

Nakata T, Hirokawa N (2003) Microtubules provide directional cues for polarized axonal transport through interaction with kinesin motor head. J Cell Biol 162:1045-1055

Nakata T, Terada S, Hirokawa N (1998) Visualization of the dynamics of synaptic vesicle and plasma membrane proteins in living axons. J Cell Biol 140:659-674 
Nilsson T, Rabouille C, Hui N, Watson R, Warren G (1996) The role of the membrane-spanning domain and stalk region of $\mathrm{N}$-acetylglucosaminyltransferase I in retention, kin recognition and structural maintenance of the Golgi apparatus in HeLa cells. J Cell Sci 109:1975-1989

Noda Y, Okada Y, Saito N, Setou M, Xu Y, Zhang Z, Hirokawa N (2001) KIFC3, a microtubule minus end-directed motor for the apical transport of annexin XIIIb-associated Tritoninsoluble membranes. J Cell Biol 155:77-88

Pavelka M, Ellinger A, Debbage P, Loewe C, Vetterlein M, Roth J (1998) Endocytic routes to the Golgi apparatus. Histochem Cell Biol 109:555-570

Polishchuk RS, Polishchuk EV, Marra P, Buccione R, Alberti S, Luini A, Mironov AA (2000) GFP-based correlative light-electron microscopy reveals the saccular-tubular ultrastructure of carriers in transit from the Golgi apparatus to the plasma membrane. J Cell Biol 148:45-58

Polishchuk EV, Di Pentima A, Luini A, Polishchuk RS (2003) Mechanism of constitutive export from the golgi: bulk flow via the formation, protrusion, and en bloc cleavage of large trans golgi network tubular domains. Mol Biol Cell 14:4470-4485

Polishchuk RS, Di Pentima A, Lippincott-Schwartz J (2004) Delivery of raft-associated, GPI-anchored proteins to the apical surface of polarized MDCK cells by a transcytotic pathway. Nat Cell Biol 6:297-307

Polishchuk RS, San Pietro E, Di Pentima A, Teté S, Bonifacino JS (2006) Ultrastructure of long range transport carriers moving from the trans Golgi network to peripheral endosomes. Traffic 7:1092-1103

Puertollano R, Martínez-Menárguez JA, Batista A, Ballesta J, Alonso MA (2001) An intact dilysine-like motif in the carboxyl terminus of MAL is required for normal apical transport of the influenza virus hemagglutinin cargo protein in epithelial Madin-Darby canine kidney cells. Mol Biol Cell 12:1869-1883

Puertollano R, van der Wel NN, Greene LE, Eisenberg E, Peters PJ, Bonifacino JS (2003) Morphology and dynamics of clathrin/ GGA1-coated carriers budding from the trans-Golgi network. Mol Biol Cell 14:1545-1557

Rindler MJ, Ivanov IE, Sabatini DD (1987) Microtubule-acting drugs lead to the nonpolarized delivery of the influenza hemagglutinin to the cell surface of polarized Madin-Darby canine kidney cells. J Cell Biol 104:231-241

Rodriguez-Boulan E, Kreitzer G, Müsch A (2005) Organization of vesicular trafficking in epithelia. Nat Rev Mol Cell Biol 6:233-247

Roth J, Taatjes DJ, Lucocq JM, Weinstein J, Paulson JC (1985) Demonstration of an extensive trans-tubular network continuous with the Golgi apparatus stack that may function in glycosylation. Cell 43:287-295

Roux A, Cappello G, Cartaud J, Prost J, Goud B, Bassereau P (2002) A minimal system allowing tubulation with molecular motors pulling on giant liposomes. Proc Natl Acad Sci USA 99:53945399
Roux A, Uyhazi K, Frost A, De Camilli P (2006) GTP-dependent twisting of dynamin implicates constriction and tension in membrane fission. Nature 441:528-531

Sahlender DA, Roberts RC, Arden SD, Spudich G, Taylor MJ, Luzio JP, Kendrick-Jones J, Buss F (2005) Optineurin links myosin VI to the Golgi complex and is involved in Golgi organization and exocytosis. J Cell Biol 169:285-295

Schuck S, Simons K (2004) Polarized sorting in epithelial cells: raft clustering and the biogenesis of the apical membrane. J Cell Sci 117:5955-5964

Shorter J, Watson R, Giannakou ME, Clarke M, Warren G, Barr FA (1999) GRASP55, a second mammalian GRASP protein involved in the stacking of Golgi cisternae in a cell-free system. EMBO J 18:4949-4960

Stoorvogel W, Geuze HJ, Griffith JM, Strous GJ (1988) The pathways of endocytosed transferrin and secretory protein are connected in the trans-Golgi reticulum. J Cell Biol 106:1821-1829

Tai AW, Chuang JZ, Bode C, Wolfrum U, Sung CH (1999) Rhodopsin's carboxy-terminal cytoplasmic tail acts as a membrane receptor for cytoplasmic dynein by binding to the dynein light chain Tctex-1. Cell 97:877-887

Teng J, Rai T, Tanaka Y, Takei Y, Nakata T, Hirasawa M, Kulkarni $\mathrm{AB}$, Hirokawa N (2005) The KIF3 motor transports $N$-cadherin and organizes the developing neuroepithelium. Nat Cell Biol $7: 474-482$

Trucco A, Polishchuk RS, Martella O, Di Pentima A, Fusella A, Di Giandomenico D, San Pietro E, Beznoussenko GV, Polishchuk EV, Baldassarre M, Buccione R, Geerts WJ, Koster AJ, Burger KN, Mironov AA, Luini A (2004) Secretory traffic triggers the formation of tubular continuities across Golgi sub-compartments. Nat Cell Biol 6:1071-1081

van Weert AW, Geuze HJ, Stoorvogel W (1997) Heterogeneous behavior of cells with respect to induction of retrograde transport from the trans-Golgi network to the Golgi upon inhibition of the vacuolar proton pump. Eur J Cell Biol 74:417-423

Wacker I, Kaether C, Krömer A, Migala A, Almers W, Gerdes HH (1997) Microtubule-dependent transport of secretory vesicles visualized in real time with a GFP-tagged secretory protein. J Cell Sci 110:1453-1463

Warner CL, Stewart A, Luzio JP, Steel KP, Libby RT, Kendrick-Jones J, Buss F (2003) Loss of myosin VI reduces secretion and the size of the Golgi in fibroblasts from Snell's waltzer mice. EMBO J 22:569-579

White J, Keller P, Stelzer EH (2001) Spatial partitioning of secretory cargo from Golgi resident proteins in live cells. BMC Cell Biol 2:19

Yeaman C, Ayala MI, Wright JR, Bard F, Bossard C, Ang A, Maeda Y, Seufferlein T, Mellman I, Nelson WJ, Malhotra V (2004) Protein kinase $\mathrm{D}$ regulates basolateral membrane protein exit from trans-Golgi network. Nat Cell Biol 6:106-112

Yeh TY, Peretti D, Chuang JZ, Rodriguez-Boulan E, Sung CH (2006) Regulatory dissociation of Tctex-1 light chain from dynein complex is essential for the apical delivery of rhodopsin. Traffic 7:1495-1502 